\title{
The Design of Physical Distribution Trainers for Athletes
}

\author{
Shumei Liu \\ Shandong University of Science and Technology \\ Qingdao, Shandong, China \\ 707408310@qq.com
}

Guanguo Ma

Shandong University of Science and Technology

Qingdao, Shandong, China

156154682@qq.com

\author{
Kailin Feng \\ Shandong University of Science and Technology \\ Qingdao, Shandong, China \\ 767416732@qq.com
}

\begin{abstract}
In this paper, an athlete physical distribution trainer is designed to help athletes train allocation of physical in runway class sports rationally. In order to improve athletic performance, based on the analysis of overall structure and controlling system of the physical distribution trainer, this paper determines the relationship between the guide of the light sport trajectory and speed of rotation of the motor and tilt motor speed, and designs the remote devise of this system. All of these provide an authentic data for control system control law. This paper mainly is to discuss the runway class sports training theory and practice and broaden the applications of sports equipment.
\end{abstract}

Keywords: Allocation of Physical; Sport Trajectory; Motor Speed; Control Law; Sports Equipment

\section{INTRODUCTION}

In the long-distance sports, athletes not only need to have physical ability, motor skills and volitive quality, but also need physical distribution rationally, so as to ensure the physical into full play. Each athlete's physical is limited, how to use limited physical, both in the whole course of the game neither wasting extra energy nor late resulting in insufficient strength, so that to make to achieve better results in the race. Then the athlete will need to be trained at a scientific and reasonable speed, and adapt to the intensity and speed in peacetime training. But how can athletes train at scientific and reasonable speed? Coaches and athletes themselves feelings are inaccurate, so researchers need an instrument guide athletes train in set speed. A new program is provided in this paper. Over the center of the stadium, researchers install a rotate light source. The light source through the motor control swing, so colored light which spot on the runway can be set according to the speed of movement, and guidance for the athletes. Then athletes can be allocated in accordance with the best rate of physical laws, to obtain the best results. The program is innovative and practical, which provides a good guidance to competitive athletes to get good results , and for the development of sports equipment provides a good reference.

\section{THE WORKING PRINCIPLE OF THE ATHLETES PHYSICAL DISTRIBUTION TRAINER}

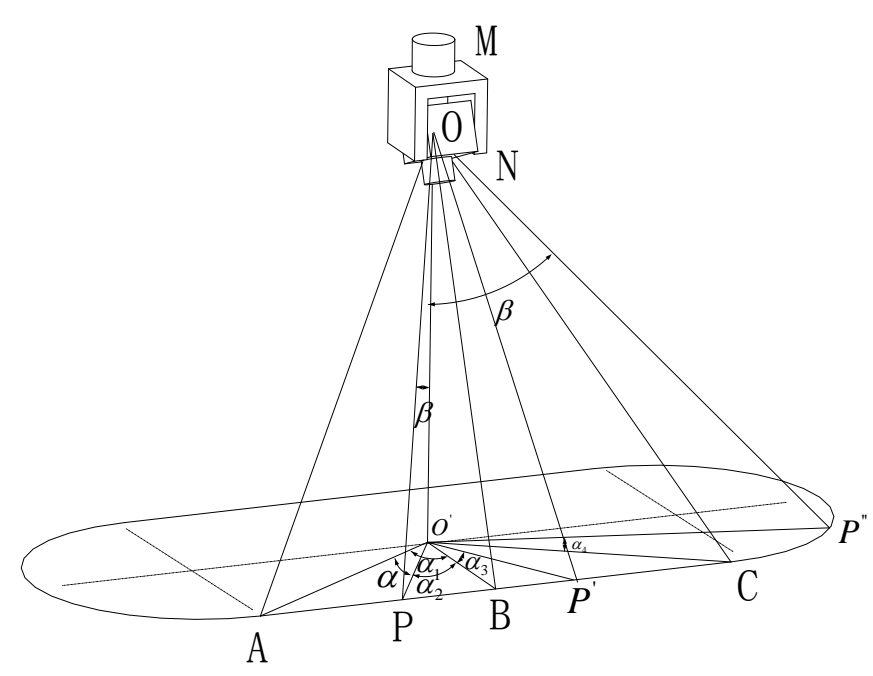

Figure 1. The Figure of the working principle of the distribution trainer

The overall structure of athletes physical distribution trainer is showed in Fig. 1. Light sources is driven by two motors. The motors can rotate around a vertical axis and bobbing up and down about a horizontal, by adjusting the speed of the two motors can be move the light spot which irradiated the ground in any track and speed. The light spot which irradiated the ground is moved at a reasonable speed. It can guide athletes in accordance with the speed training, so as to achieve a reasonable physical dis tribution effects.

\section{THE OVERALL STRUCTURE OF}

\section{ATHLETES PHYSICAL ST RENGTH DIST RIBUTION TRAINER}

As shown in Fig. 2, the overall structure of athletes physical distribution trainer include system fixed seat, rotating motor base, rotating motor, light source stand, light source, pitching motor and remote control, and other components. The trainer fixed seat is mounted above the 
center of the runway, and rotating motor base is mounted under it. The rotating motor is mounted on this fixed seat. The light source stand is mounted at the bottom of the rotating motor output shaft. The light source is mounted on the light source stand through the bearing. The pitching motor is mounted on one side of the light source stand. Both rotating motor and pitching motor have encoders on the output shaft in order to detect the rotation angle and speed of rotating motor and pitching motor. The remote control can control open, stop, reset and light spot velocity of the physical distribution trainer. In the physical distribution trainer working process, rotating motor control rotation motion of the light source, and pitching motor control pitch motion of the light source. It can be achieved that the light spot on the ground achieve movement at any speed and in any track by controlling rotation and pitch motion track of light source.
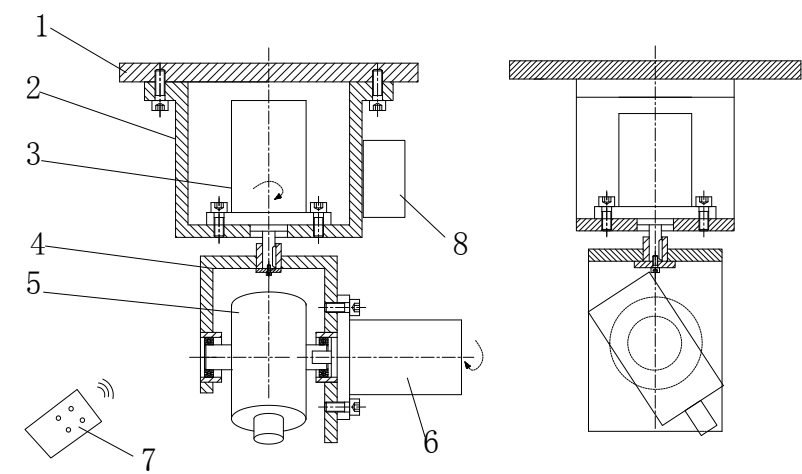

Figure 2. The overall structure of the physical distribution trainer

\section{THE DESIGN OF THE MOT OR ROT ATION RULE}

For irradiated light spot on the ground can be completed in accordance with the route set from straight to arc, then uniform motion from arc to straight line, the need to rotate the motor and tilt motor coordination can be achieved.

As shown in Fig. 1, researchers set the speed $v$ is constant motion, so can be set speed $\mathrm{v}$ to a constant and the size of the set speed can be adjusted by remote control.

When the light spot movement on a straight line segment $\mathrm{AB}$, setting $0 \leq v t<l / 2$

$$
\begin{aligned}
& \tan \alpha_{2}=\frac{B P}{O^{\prime} P}=\frac{l / 2-v t}{R} \\
& \alpha=\angle A O^{\prime} P=\alpha_{1}-\alpha_{2}=\alpha_{1}-\arctan \frac{l / 2-v t}{R} \\
& \beta=\angle P O O^{\prime}=\arctan \frac{O^{\prime} P}{O O^{\prime}}=\arctan \frac{\sqrt{(l / 2-v t)^{2}+R^{2}}}{h}
\end{aligned}
$$

In this equation:

Point $\mathrm{P}$ is the straight line motion light point on any point, then $A P=v t, B P=l / 2-v t$

$l \_$Runway length line segment.

When the light spot movement in a straight line segment BC, setting $l / 2 \leq v t<l$,
(1)

$$
\tan \alpha_{3}=\frac{B P^{\prime}}{O^{\prime} B}=\frac{v t-l / 2}{R}
$$

$$
\alpha=\angle A O^{\prime} P^{\prime}=\alpha_{1}+\alpha_{3}=\alpha_{1}+\arctan \frac{v t-l / 2}{R}
$$

(2)

$$
\begin{aligned}
& \beta=\angle P^{\prime} O O^{\prime}=\arctan \frac{O^{\prime} P^{\prime}}{O O^{\prime}} \\
& =\arctan \frac{\sqrt{(v t-l / 2)^{2}+R^{2}}}{h}
\end{aligned}
$$

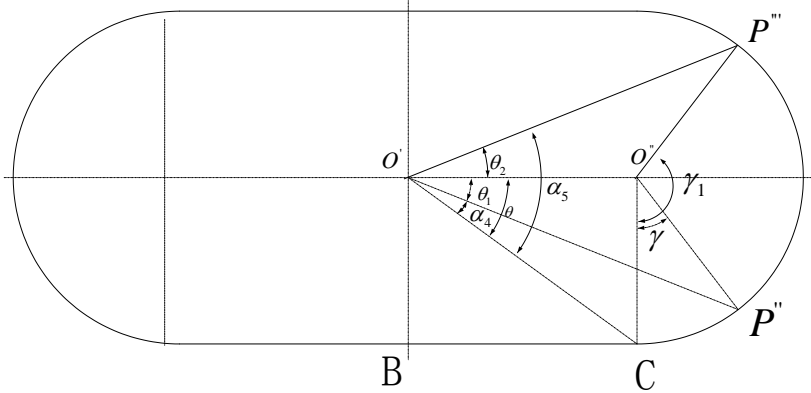

Figure 3. The geometric sketch of the track plane

As for Fig. 3, in the curve section, when $0<\gamma<\pi / 2$

$$
\begin{aligned}
& O^{\prime} P^{\prime \prime}=\sqrt{(l / 2)^{2}+R^{2}-2 \times(l / 2) \times R \times \cos (\pi / 2+\gamma)} \\
& =\sqrt{l^{2} / 4+\mathrm{R}^{2}+l R \sin \gamma}
\end{aligned}
$$

(4)

$$
\begin{aligned}
& \theta_{1}=\arccos \left[\frac{\left(O^{\prime} O^{\prime \prime}\right)^{2}+\left(O^{\prime} P^{\prime \prime}\right)^{2}-\left(O^{\prime} P^{\prime \prime}\right)^{2}}{2\left(O^{\prime} O^{\prime \prime}\right) \times\left(O^{\prime} P^{\prime \prime}\right)}\right] \\
& =\arccos \left(\frac{l^{2}+2 l R \cos \gamma}{2 l \times \sqrt{l^{2} / 4+\mathrm{R}^{2}+l R \sin \gamma}}\right)
\end{aligned}
$$

(5)

$$
\begin{aligned}
& \alpha=\angle A O^{\prime} P^{\prime \prime}=2 \alpha_{1}+\alpha_{4} \\
& =2 \alpha_{1}+\arctan \frac{2 R}{l}-\arccos \left(\frac{l^{2}+2 l R \cos \gamma}{2 l \times \sqrt{l^{2} / 4+\mathrm{R}^{2}+l R \sin \gamma}}\right)
\end{aligned}
$$

(6)

$\beta=\angle P^{\prime \prime} O O^{\prime}=\arctan \frac{O^{\prime} P^{\prime \prime}}{O O^{\prime}}=\arctan \frac{\sqrt{l^{2} / 4+\mathrm{R}^{2}+l R \sin \gamma}}{h}$

In this equation:

$$
\angle O^{\prime} O^{\prime \prime} P^{\prime \prime}=\pi / 2+\gamma=\pi / 2+\frac{v t-l}{R}
$$

when $\pi / 2<\gamma<\pi$, 


$$
\begin{aligned}
& \theta_{2}=\arccos \left[\frac{\left(O^{\prime} O^{\prime \prime}\right)^{2}+\left(O^{\prime} P^{\prime \prime \prime}\right)^{2}-\left(O^{\prime \prime} P^{\prime \prime \prime}\right)^{2}}{2\left(O^{\prime} O^{\prime \prime}\right) \times\left(O^{\prime} P^{\prime \prime}\right)}\right] \\
& =\arccos \left(\frac{l^{2}+2 l R \sin \gamma_{1}}{2 l \times \sqrt{l^{2} / 4+\mathrm{R}^{2}+l R \sin \gamma_{1}}}\right) \\
& \alpha=\angle A O^{\prime} P^{\prime \prime}=2 \alpha_{1}+\alpha_{4} \\
& =2 \alpha_{1}+\arctan \frac{2 R}{l}+\arccos \left(\frac{l^{2}+2 l R \sin \gamma_{1}}{2 l \times \sqrt{l^{2} / 4+\mathrm{R}^{2}+l R \sin \gamma_{1}}}\right) \\
& \beta=\angle P^{\prime \prime} O O^{\prime}=\arctan \frac{O^{\prime} P^{\prime \prime}}{O O^{\prime}} \\
& =\arccos \frac{\sqrt{l^{2} / 4+\mathrm{R}^{2}+l R \sin \gamma_{1}}}{h}
\end{aligned}
$$

\section{THE DESIGN OF ATHLETES PHYSICAL STRENGTH DISTRIBUTION TRAINER SYSTEM}

\section{A. The selection of the control system}

According to the rotating law of these two motors, researchers can see that the track and speed of the guiding spot have a strict relaction with the rotate speed of ratating motor and swing motor. Firstly, in order to ensure that the guiding spot can runs with a setting track and stabilized velocity, the speed of these two motors must be stabilized, so that the unstable phenomenon of the guiding spot can't take place because of the factors such as inertia and external disturbance, which can cause the excursion of the guiding spot. Then in order to ensure that athletes can distribute the strength better in the progress of exercise, researchers also need the guidance spot can run stably or can accelerate and decelerate according to the setting speed ahead of time.

In the open-loop control system, the error may be the unstable, because these two motors rotate without relevance between each other and have no self-checking module, which can make the guiding of the physical distribution trainer unstable. And in the closed-loop control system, these two motors are under self-inspection and self-regulation in every time. And in this system, that the rotating motor and swing motor runs with good cooperation can be ensured, in which the guiding spot can runs with the setting track and velocity. Therefore, researchers use the closed-loop control system in this control system of the physical distribution trainer, and the speed regulator is introduced into the system. Then the speed feedback control loop is made up. The control sketch of the speed feedback control loop is showed as Fig. 4. In this speed feedback control loop the PID regulator is used to deal with the relevant deviation, in order to acquire the better static characteristic and dynamic characteristic. And the deviation is put into the CPU. The CPU manages these deviations in a united way, and puts out the PWM signal to control these two motors.

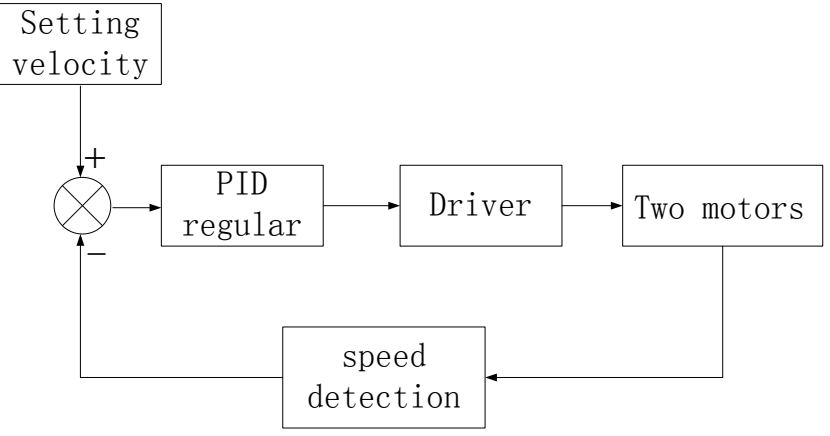

Figure 4. The control sketch of the speed feedback control loop

\section{B. The construction of the control system}

As shown in Fig. 5, the software structure of athletes physical distribution trainer include CPU, data acquisition module, encoder, rotating motor drive module and pitching motor drive module, remote control module and other components. The encoder is an incremental encoder to convert the motor turned angle into periodic electrical signal and to convert this electrical signal into count pulse. The number of pulses represents the angle of the motor rotation. Data acquisition module collects count pulse that encoder generated and inputs the pulse signal to the CPU. The CPU deals with the signal according to the set procedure uniformly and gives the orders to control the rotation speed of rotating motor and pitching motor. The CPU is based on the STM32 singlechip.It analyzes faster. The remote control module with miniscope is used because of the need to control open, stop, reset and velocity of the instrument on the ground.

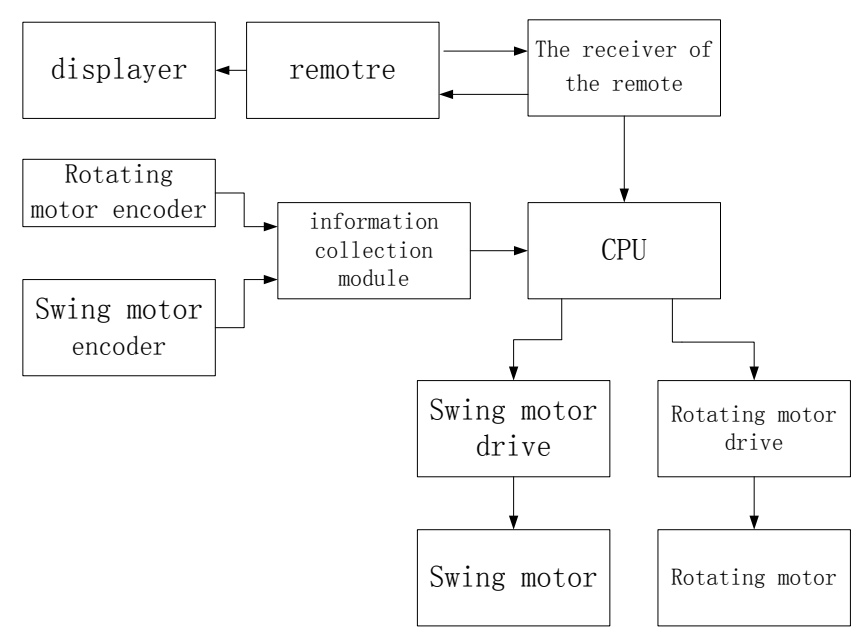

Figure 5. The control system of athletes physical strength distribution trainer

\section{THE DESIGN OF THE REMOTE CONTROL MODULE}

Along with the development of the electronic technology, the wireless remote control technology has a great development, and its intelligence is also significantly improved. Because the wavelength of infrared remote control is much smaller than the wavelength of the radio, it is not easy for the infrared remote control to disturb the 
normal work of other appliances, which is its unique advancement, compared with other way. Meanwhile this remote way has a lower working voltage, smaller power and simple peripheral circuit, so infrared remote control module is used in this system.

The infrared remote controlled system mainly concludes emitter and receiver, to operate and control by modulating and demodulating circuit.
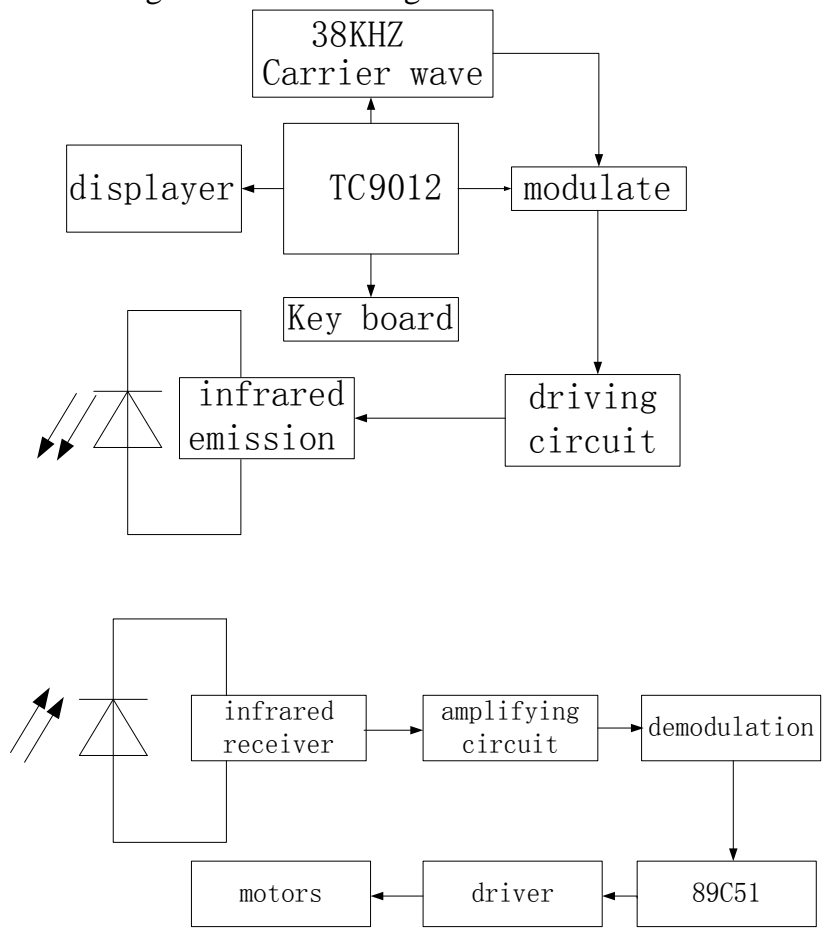

Figure 6. The block diagram of the remote control module

The design of the remote control module can be described as Fig. 6. The pulse signal is coded modulated, then to drive the infrared light emitting diode to give out infrared wave after modulating. The common carrier frequency is $38 \mathrm{KHZ}$. And the setting infrared receiver circuit uses the HSO038 that is off-the-shelf. The HS0038 integrates the function of infrared receiving, amplification and demodulation. The center frequency is $38 \mathrm{KHZ}$. The display module is 12864 liquid crystal display carrying word stock. This display mainly has 3 ports. These ports respectively connect to P1.4, P1.5 and P1.6 on the CSM. There are 5 buttons on the keyboard of the remote. These buttons have the functions including speed increase, speed decrease, starting, stopping and reset. Besides, the speed increasing and speed decreasing is incremental.

\section{CONCLUSIONS}

The light source rotation and the pitch swing can make the colored light spot irradiated on the runway move in accordance with the set speed and track. The athletes can make a reasonable distribution of speed and power according to the guidance of the light spot. Using the remote control can control open, stop, reset of the instrument and change the light spot velocity. The remote control display can show the light spot velocity. In this paper, researchers studied the hardware structure and the software structure of the instrument, and studied the relationship between the movement rule of the two motors and the track and speed of the light spot to draw relevant laws. The infrared remote control module is designed. It provided a theoretical basis for the development of equipment.

\section{REFERENCES}

[1] Yu Baihui. The introduction of the distributing strength in middlelong-dist ancerace[J]. Exchange of experience, 2014(6), 120-121.

[2] Pu lianggui, Ji Minggang. Design of Mechinery[M]. Beijing, Higher Education Press, 2003.

[3] Cheng Daxian. Hand Book of Mechanical Design[K].Beijing, Chemical Industry Press, 2004.

[4] Wang Junfeng, Xue Hongde. Modern control technology and application[M].Beijing: Posts \& Telecom Press, 2005.

[5] Li Tiantian, Chen Hong, Li Jingtao. Feedback Control of DC Motor by PID Algorithm[J]. Electro-Optic Technology Application, 2009(12),55-57.

[6] Sun Yuanwen. The DC motor control based on the MCU[J]. Science \&Technology information, 2010(35),1154-1155.

[7] Zhu Lisheng. A survey on DC motor closed- loop control technology[D]. Nanjng: Nanjing University of Science and Technology, 2009.

[8] Du Yingcai, Wang Xijun, Wang Shujie, and so on. Auto-detection system of incremental encoder[J]. Journal of Electronic Measurement and Instrument, 2012(11),993-997.

[9] Bao Song, Bao Kejin, Yu Jinghua. Measurement and Control System of Direct Current Electromotor Based on PID and SCM[J]. Microcomputer Development, 2003(8), 72-74.

[10] Tong Qiangmin. A sort of Wireless Romote Control Technology Based SCM System[J]. Computer Knowledge and Technology, 2009(3), 2021-2022.

[11] Zhang Hongtao, Yan Lin, Xu Xiaohui, and so on. Research on Brushless DC Motor Control System Based on PID and SCM[J]. Modern Electronic Technology, 2007(10),52-54.

[12] Gui Wenbin. Design of dual closed-loop control system based on DSP for DC motor

Corresponding author: Feng Kai-lin; TEL:13969849679; Email:156154682@qq.com 